\title{
PROPOSTA DE HEURÍSTICA DE SEQUENCIAMENTO PARA RETOMADA DE PILHAS DE MINÉRIO EM PÁTIOS DE ESTOCAGEM*
}

Fabiano Della Libera da Silva ${ }^{1}$

\section{Resumo}

Este artigo propõe uma heurística de sequenciamento para a retomada de pilhas de minério (entendidas como tarefas a serem sequenciadas) nos seus respectivos pátios através de um índice de priorização de pilhas (IP). Tal índice apoia-se em fatores importantes para as operações de pátios de estocagem, como capacidade das recuperadoras (máquinas), qualidade do minério, tempo de residência e tempo de deslocamento das recuperadoras entre pilhas (setup). O método proposto foi aplicado em dois pátios, duas máquinas recuperadoras e doze pilhas e a sequência de recuperação sugerida pelo método foi considerada coerente por especialistas de processo da empresa mineradora.

Palavras-chave: Heurística; Sequenciamento; Pátios de estocagem.

\section{PROPOSAL OF HEURISTIC OF SEQUENCING FOR RECLAIMING ORE PILES IN STOCKYARDS}

\section{Abstract}

This work aims to propose a heuristic for sequencing reclaiming ore piles (seen as tasks to be sequenced) in their respective stockyard by applying a pile prioritization index (IP). This index rely on relevant factors to the stockyard operation, including capacity of reclaimers (machines), ore quality, time of ore residence, and travel time of reclaimers between piles (setup). The main contribution relied on the proposition of a new index for sequencing pile recovering that avoids the use of subjective rules-ofthumb typically applied in the sector. The proposed heuristic yielded satisfactory results when assessed by process experts.

Keywords: Heuristics; Scheduling; Stockyards.

1 Engenheiro de Produção Civil, Mestrado Profissional em Produção Mineral, PPGE3M, UFRGS, Porto Alegre, RS, Brasil. 


\section{INTRODUÇÃO}

Este artigo propõe uma heurística de sequenciamento baseada em um índice de priorização de pilhas (IP) a serem recuperadas com base em fatores relevantes para o processo de carregamento, os quais incluem a capacidade das máquinas recuperadoras, qualidade do minério a ser carregado e transportado e tempo de deslocamento entre pilhas. Nos processos hoje existentes, a tomada de decisão é feita, normalmente, pelo técnico de controle de qualidade e/ou pelo técnico de operações de pátio, fazendo com que a mesma seja de forma subjetiva e sem base acadêmica. Para tanto, a heurística proposta inicialmente avalia a capacidade das máquinas e o número de pilhas a serem recuperadas. Em seguida, as pilhas são atribuídas às máquinas de acordo com suas capacidades. Na sequência, gera-se um índice de priorização de recuperação de cada pilha (que estabelece a sequência das pilhas a serem recuperadas), o qual inicia pelo cálculo do índice de qualidade de cada pilha (Qi). Para cada uma das pilhas, deriva-se um critério (parâmetro) de qualidade e, através da aplicação do fator perda de Taguchi [1], calcula-se o OQE Overall Quality of Production Effectiveness (Qualidade Global Efetiva), onde cada pilha recebe uma nota proporcional à sua qualidade. Na sequência, gera-se uma matriz de setup contemplando os tempos de trânsito da recuperadora entre as pilhas; tal matriz também comporta restrições físicas e dificuldade de locomoção das máquinas recuperadoras entre as pilhas a serem recolhidas. $O$ índice obtido, IP, determina a sequência de recuperação das pilhas.

\section{FUNDAMENTAÇÃO TEÓRICA}

\subsection{Conceitos Básicos de Sequenciamento}

Davis et al. [2] e define sequenciamento como a determinação das máquinas ou centros de trabalho a serem utilizados no processamento de uma determinada tarefa. Técnicas de sequenciamento são bastante difundidas em organizações industriais, sendo aplicadas no apoio aos processos decisórios através da alocação de recursos e tarefas. Além disso, tais técnicas permitem definir uma ordem apropriada para execução de tarefas de acordo com restrições específicas.

De acordo com Pinedo [3], um problema de sequenciamento pode ser descrito no formato $\alpha|\beta| \gamma$, onde $\alpha$ descreve o ambiente da máquina, o campo $\beta$ fornece detalhes de características de processamento e as restrições, e o campo y descreve o objetivo a ser otimizado.

Sistemáticas para sequenciamento de tarefas encontram vasta aplicação em diversos setores produtivos, incluindo cenários de extração em mina.

Em sequenciamento aplicado à mineração, procura-se ainda determinar a sequência ideal em que o material deve ser lavrado durante a vida útil de uma mina a fim de maximizar o valor presente líquido de suas operações. Com esta finalidade, Bley et al [4] desenvolveram uma formulação de programação inteira utilizando restrições de precedência e de produção identificando variáveis que podem ser resolvidas antes da otimização, reduzindo significativamente o número de variáveis na formulação do problema e, consequentemente, nos requisitos de cálculo e processamento computacional. Semelhantemente, Boland et al [5] sustentam que uma sequência adequada de lavra de blocos discretizados de uma mina traz ganhos significativos nas esferas de planejamento, programação da produção, otimização de processos, 
garantia da qualidade, sustentabilidade do processo de lavra e também na redução de custos em todo o processo da mina.

\subsection{Critérios Relevantes para o Sequenciamento em Pátios de Recuperação de Minério}

É muito importante a escolha de critérios adequados para sequenciamento das pilhas nos pátios de forma que seja gerado o melhor aproveitamento dos recursos produtivos, níveis adequados na quantidade de minério nos pátios e aumento da taxa de retirada dos estoques. Alguns critérios relevantes para tal fim são detalhados na sequência.

\subsubsection{Qualidade do minério}

Minas de extração normalmente utilizam o indicador OQE para medição da qualidade dos seus produtos. O OQE é aplicado nos processos desde a mina até 0 porto, apoiando-se na função-perda de Taguchi (Loss Function). A função de Taguchi, vastamente utilizada em cenários de engenharia, utiliza uma combinação de métodos estatísticos para alcançar melhorias rápidas na qualidade e no custo através da otimização do design do produto e do processo de produção. A função perda de Taguchi ainda estabelece uma medida financeira para o cálculo do desvio de uma característica do produto com relação ao valor nominal conforme $\mathrm{L}=$ $\mathrm{k}(\mathrm{y}-\mathrm{T})^{2}$, onde $\mathrm{L}$ representa a perda devido ao desvio da característica; $\mathrm{k} o$ coeficiente de perda; y o valor da característica de qualidade; e T o valor nominal ou valor alvo.

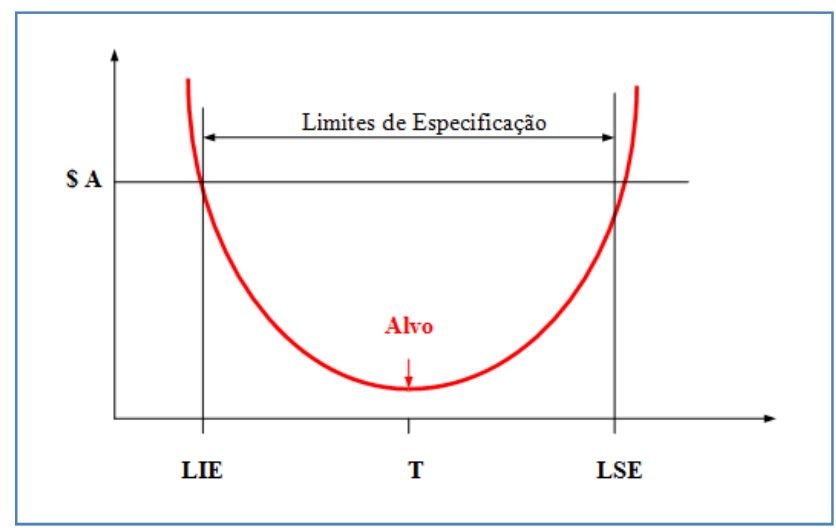

Figura 1 - Função perda de Taguchi [6]

\subsubsection{Tempo de residência do minério}

A liberação de água (umidade) de uma pilha de minério é um critério fundamental em pátios de estocagem de minério, pois pode influenciar no processo de beneficiamento (visto que, em alguns destes processos, a umidade poderá elevar-se ainda mais devido à adição de água). Dronkers e Zimmerman [7] definem tempo de residência como o tempo que a pilha aguarda no pátio a partir de sua formação até o seu carregamento para que uma parcela da água existente na pilha escorra. Tempos de residência reduzidos faz com que os níveis de umidade sejam elevados por deficiência na drenagem, contribuindo para a compactação do minério durante o seu transporte na ferrovia. 


\subsubsection{Tempo de mudança de pilha (setup)}

Os tempos de setup compreendem o tempo de preparação de uma máquina para processar a tarefa seguinte, o qual inclui operações de agrupamento de ferramentas necessárias e limpeza de máquina, entre outras situações [8].

A retomadora de minério (recuperadora) é instalada nos pátios de estocagem e movimentam-se no sentido longitudinal aos mesmos (movimento de translação) e podem girar num ângulo de $180^{\circ}$ (movimento de giro), fazendo com que esta possa recuperar pilhas em dois pátios paralelos. Tal tempo de trânsito entre a pilha que acaba de ser recuperada e a próxima a ser removida caracteriza o tempo de setup em uma mina.

\subsubsection{Balanceamento dos pátios}

Em um sistema de pátios, é desejável que a distribuição de massa das pilhas nas pilhas seja uniforme, de maneira a reduzir a necessidade de deslocamento da recuperadora para encontrar uma nova pilha dentro das características desejadas. Um balanceamento adequado de massa também facilita situações onde existam restrições de movimentação geradas por manutenção no pátio ou quebra de outra recuperadora no mesmo trilho (normalmente devido à quebra ou manutenção nos trilhos ou de outro equipamento existente no sistema) [9].

\section{MÉTODO}

\subsection{Verificação das Máquinas e Pilhas a Serem Sequenciadas}

Considere abaixo um sistema genérico composto por dois pátios, A e B, com duas máquinas (M1 e M2) aptas a recuperarem pilhas de ambos os pátios (ilustrado na Figura 2). As pilhas pertencentes ao pátio $\mathrm{A}$ são identificadas por $P_{A} 1, P_{A} 2, P_{A} 3, \ldots, P_{A} i$, enquanto que as pilhas pertencentes ao pátio $\mathrm{B}$ são descritas por $P_{B} 1, P_{B} 2, P_{B} 3, \ldots, P_{B} j$; $m$ é a massa existente em cada pilha. $O$ método proposto pode ser estendido a qualquer número de máquinas e pilhas.

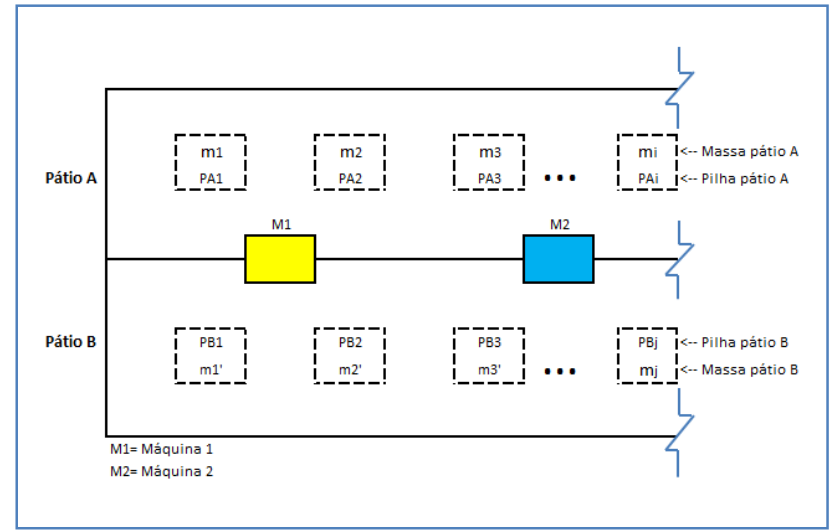

Figura 2 - Representação esquemática dos pátios, pilhas e recuperadoras

\subsection{Alocação das Pilhas às Máquinas em Função de suas Capacidades}

Considere as capacidades das máquinas M1 e M2 como CapM1 e CapM2, respectivamente; o número total de pilhas nos pátios $\mathrm{A}$ e $\mathrm{B}$ é $n$. O número de pilhas a serem alocadas às máquinas M1 e M2, QM1 e QM2, respectivamente, é definido pelas equações (1) e (2). 


$$
Q M 1=n \cdot\left[\frac{\text { CapM } 1}{\operatorname{CapM} 1+\operatorname{CapM} 2}\right] \quad \text { (1) } Q M 2=n \cdot\left[\frac{\text { CapM2 }}{\operatorname{CapM} 1+C a p M 2}\right]
$$

$\mathrm{Na}$ sequência, as pilhas são alocadas às máquinas recuperadoras. Para a escolha das pilhas, as máquinas partirão de uma posição inicial nos extremos do pátio, movendo-se em direção ao centro do pátio e varrendo o número de pilhas definido pelas equações (1) e (2), conforme ilustrado na Figura 3.

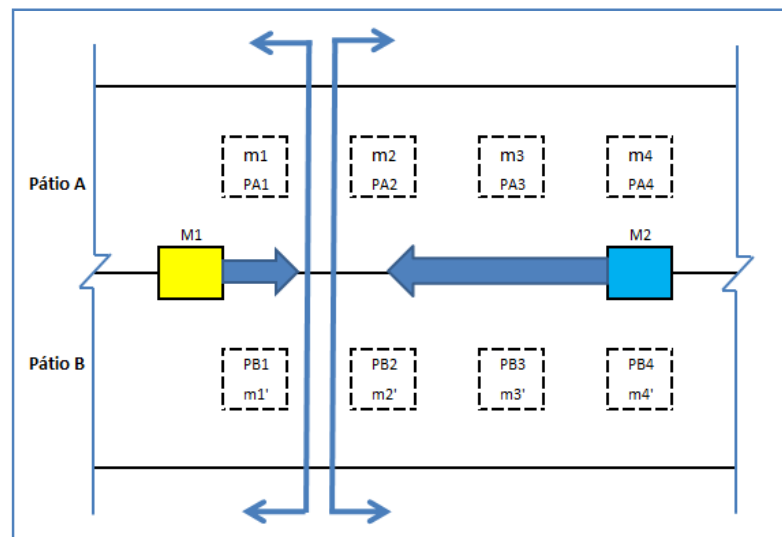

Figura 3 - Alocação das pilhas no pátio às máquinas M1 e M2

Na sequência, com a aplicação da equação (3), verifica-se o balanceamento das pilhas alocadas a cada máquina. No exemplo genérico da Figura 3, as pilhas $P_{A} 1$ e $P_{B} 1$ são direcionadas à máquina $\mathrm{M} 1$ e as pilhas $P_{A} 2, P_{A} 3, P_{A} 4, P_{B} 2, P_{B} 3$ e $P_{B} 4$, à máquina M2. A quantidade de pilhas para cada máquina dependerá tanto do tamanho das pilhas (massa das pilhas) quanto da capacidade das recuperadoras.

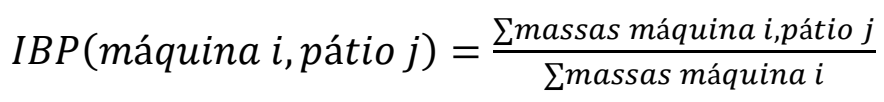

O quadro 1 sumariza as equações para o balanceamento em dois pátios (A e $B)$ e duas máquinas (M1 e M2).

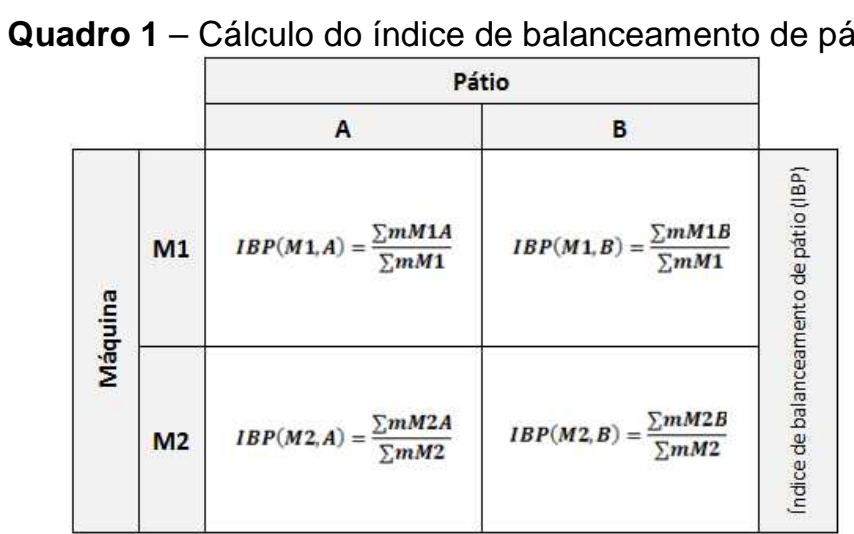

onde

$\sum m M 1 A$ é a soma das massas das pilhas que pertencem a Máquina $\mathrm{M} 1$ e ao pátio $\mathrm{A}$; $\sum m M 1 B$ é a soma das massas das pilhas que pertencem a $\mathrm{M} 1$ e ao pátio B; $\sum m M 1$ é a soma das massas das pilhas que pertencem a M1; $\sum m M 2 A$ é a soma das massas das pilhas que pertencem a $M 2$ e ao pátio $A ; \sum m M 2 B$ é a soma das massas das pilhas que pertencem a M2 e ao pátio B; e $\sum m M 2$ é a soma das massas das pilhas que pertencem a M2. 


\subsection{Cálculo do Índice de Qualidade das Pilhas $(Q i)$}

Nesta etapa, os índices de qualidade das pilhas são calculados através das equações (4) e (5), as quais são adaptadas da função perda de Taguchi

$$
Q i=O Q E=10-K(\text { Meta }-x i)^{2} \quad \text { (4) } K=\frac{10}{(\text { Meta-Limite })^{2}}
$$

Onde K é o coeficiente de perda; Meta é a meta da especificação do parâmetro; e Limite são os limites inferior ou superior (LIE ou LSE) de qualidade do parâmetro.

Para o cálculo do índice de qualidade, serão considerados somente valores maiores ou iguais a 1, justificado pelo fato deste valor participar da equação do índice de prioridade como um elemento de multiplicação (sendo o número 1 um elemento neutro). Portanto, valores de OQE menores que 1 serão considerados iguais a 1 .

\subsection{Construção da Matriz de Tempo de Mudança de Pilha (Matriz Setup)}

A matriz de setup avalia as potenciais mudanças entre pilhas e seus respectivos tempos $\left(S_{i}\right)$, conforme ilustrado na Figura 4. Tais dados são extraídos de medições diretas de tempo de movimentação de máquinas entre pilhas.

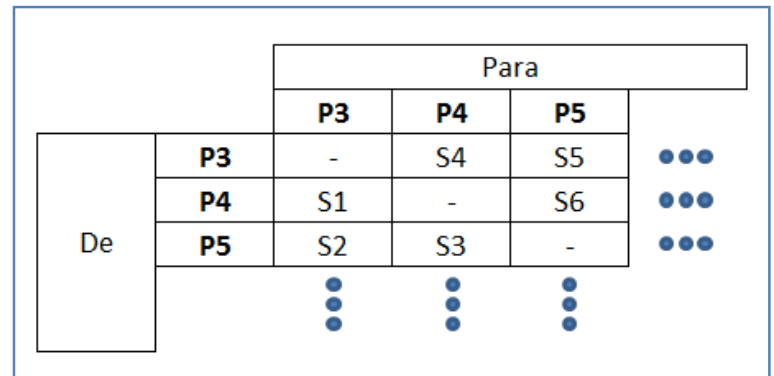

Figura 4 - Matriz setup com os valores dos tempos $\left(S_{i}\right)$

\subsection{Obtenção dos Tempos de Residência das Pilhas $\left(T_{i}\right)$}

Os tempos de residência $T_{i}$ são estimados em função dos horários de início e término da formação de uma pilha, ou seja, é o tempo compreendido entre o início do empilhamento do minério no pátio em uma determinada pilha até o início da recuperação desta pilha. Sistemas produtivos com tecnologia Enterprise Resource Planning (ERP) fazem a coleta constante destes valores, facilitando sua obtenção.

\subsection{Cálculo do Índice de Priorização de Pilha (IP)}

A equação (6) abaixo traz o índice de priorização da pilha $i$ (IP $i$ ) proposto, o qual permite definir a ordem de recuperação das pilhas.

$$
I P i=Q i \cdot T i \cdot \frac{1}{S i} \cdot I B P i
$$

Onde $Q i$ é o índice de qualidade de cada pilha; $T i$ é o tempo de residência de cada pilha; Si é o tempo de mudança entre a pilha atual em recuperação até a pilha $i$ (tempo de setup); e IBPi é o índice de balanceamento de pátios. 


\section{APLICAÇÃO DA SISTEMÁTICA EM UM SISTEMA DE RECUPERAÇÃO DE MINÉRIO}

A sistemática proposta foi aplicada na mina de Carajás, a qual iniciou suas operações de extração em 1985 e é considerada a maior mina de ferro a céu aberto no mundo, tendo alcançado marca de um bilhão de toneladas produzidas em 2007; sua produção anual é de aproximadamente 120 milhões de toneladas métricas [10]. O método foi aplicado em um sistema composto por dois pátios (A e B), com duas máquinas recuperadoras (M1 e M2) e doze pilhas (sendo cada pilha representada por $P A j$ e $P B j$, onde $A$ e $B=$ pátios e $j=$ número da pilha). As recuperadoras (M1 e M2) apresentam capacidades de 6.000 ton./h e 8.000 ton./h, respectivamente. A Figura 5 ilustra esta configuração, especificando o volume de minério em cada pilha (massas em kt [×1.000]) antes do início da etapa de recuperação.

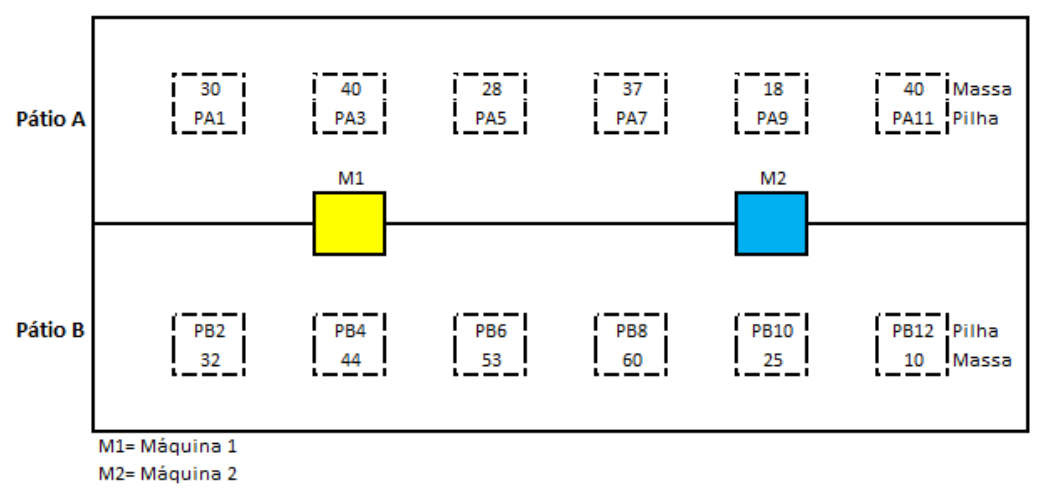

Figura 5 - Representação dos pátios, pilhas e suas massas e recuperadoras.

As equações (1) e (2) permitem determinar o número de pilhas a serem alocadas a cada máquina, como segue.

$$
\begin{aligned}
& Q M 1=n \cdot\left[\frac{\text { CapM } 1}{\text { CapM } 1+\text { CapM2 } 2}\right]=12 \cdot\left[\frac{6.000}{6.000+8.000}\right]=5,14 \sim 5 \text { pilhas } \\
& Q M 2=n \cdot\left[\frac{\text { CapM2 }}{\text { CapM } 1+\text { CapM2 } 2}\right]=12 \cdot\left[\frac{8.000}{6.000+8.000}\right]=6,86 \sim 7 \text { pilhas }
\end{aligned}
$$

Atribuindo-se as pilhas às máquinas M1 e M2 de acordo com suas distâncias iniciais das máquinas, obtém-se o cenário da Figura 6.

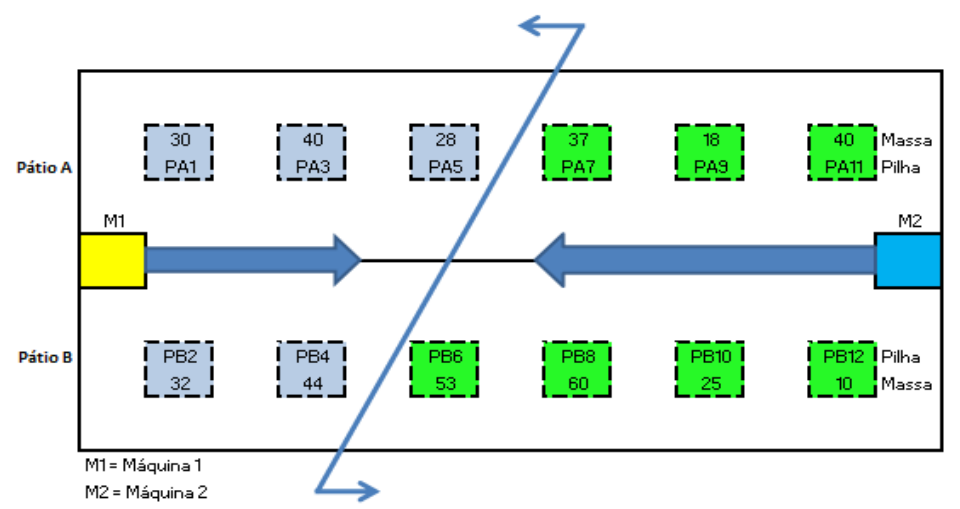

Figura 6 - Representação das pilhas e as suas respectivas máquinas.

Em função das suas posições nos pátios, as pilhas $P_{A} 5$ e $P_{B} 6$ poderiam ter sido alocadas tanto para M1 quanto para M2. Conforme sugerido em (2.3.2), a pilha com 
maior massa $\left(P_{B} 6=53 \mathrm{kt}\right)$ foi alocada para a máquina de maior capacidade $(\mathrm{M} 2=$ 8.000 ton/h.). A equação (3) permite estimar a distribuição (balanceamento) das máquinas e pátios, como exemplificado para a Máquina 1 e Pátio $A$.

$\operatorname{IBP}(M 1, A)=\frac{\sum m M 1 A}{\sum m M 1}=\frac{m 1+m 3+m 5}{m 1+m 3+m 5+m 2+m 4}=\frac{30+40+28}{30+40+28+32+44} \cong 0,563$

A Tabela 1 sumariza os valores dos balanceamentos dos pátios para as máquinas e seus respectivos pátios.

Tabela 1 - Índice de balanceamento de pátio (IBP) por quadrante

\begin{tabular}{|c|c|c|c|c|}
\hline & \multicolumn{2}{|c|}{ Pátio } & \\
\hline & & A & B & \multirow{3}{*}{ 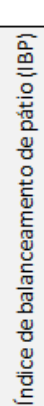 } \\
\hline \multirow{2}{*}{ 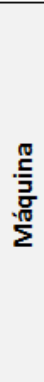 } & M1 & $\begin{array}{c}\text { IBP }=0,563 \\
\text { Pilhas P1, P3 e P5 }\end{array}$ & $\begin{array}{l}\text { IBP }=0,437 \\
\text { Pilhas P2 e P4 }\end{array}$ & \\
\hline & M2 & $\begin{array}{c}\text { IBP }=0,391 \\
\text { Pilhas P7, P9 e P11 }\end{array}$ & $\begin{array}{c}\text { IBP }=0,609 \\
\text { Pilhas P6, P8, P10 e P12 }\end{array}$ & \\
\hline
\end{tabular}

Na sequência, o percentual de ferro (\%Fe) das pilhas no pátio é utilizado para o cálculo do índice de qualidade das mesmas, conforme a Tabela 2.

Tabela 2 - Teor de ferro das pilhas no pátio

\begin{tabular}{|c|c|c|c|}
\hline Pilha & $\angle \mathrm{FE}$ & Pilha & $\not \mathrm{Fe}$ \\
\hline PÁ1 & 65.25 & $\mathrm{~PB} 2$ & 64,36 \\
\hline PA3 & 65,00 & PB4 & 6399 \\
\hline PA5 & 65.40 & PE6 & 65,69 \\
\hline PA7 & 61.45 & PBG & 64.25 \\
\hline PA9 & 64,10 & PE10 & 63.48 \\
\hline PA11 & 62,12 & PB12 & 66,16 \\
\hline
\end{tabular}

Considerando-se as especificações de qualidade vigentes atualmente na mina (Meta = 64,30; $\mathrm{LIE}=63,30$ e LSE = 65,30), obtém-se $\mathrm{K}$ e os índices de qualidade apresentados na Tabela 3 utilizando-se as equações (4) e (5).

$$
\begin{gathered}
K=\frac{10}{(\text { Meta }- \text { Limite })^{2}}=\frac{10}{(64,30-63,30)^{2}}=10 \\
\mathrm{Q} i=10-\mathrm{K}(\text { Meta }-\mathrm{xi})^{2}
\end{gathered}
$$

\begin{tabular}{|c|c|c|c|c|c|}
\hline Pilha & $\% \mathrm{Fe}$ & $\mathrm{Q} j$ & Pilha & $\% \mathrm{Fe}$ & $\mathrm{Q} j$ \\
\hline PA1 & 65,25 & 1 & PB2 & 64,38 & 9,94 \\
\hline PA3 & 65,00 & 5,10 & PB4 & 63,99 & 9,04 \\
\hline PA5 & 65,40 & 1 & PB6 & 65,89 & 1 \\
\hline PA7 & 61,45 & 1 & PB8 & 64,25 & 9,98 \\
\hline PA9 & 64,10 & 9,60 & PB10 & 63,48 & 3,28 \\
\hline PA11 & 62,12 & 1 & PB12 & 66,18 & 1 \\
\hline
\end{tabular}

Tabela 3 - Índice de qualidade das pilhas nos pátios

Na sequência, construiu-se uma matriz de tempo acerca do deslocamento entre pilhas (matriz setup), apresentada na Tabela 4. Tais tempos foram estimados 
levando-se em conta os movimentos de translação (longitudinal ao pátio) e rotação (giro) da recuperadora.

Tabela 4 - Matriz setup com os valores dos tempos em minutos (SI).

\begin{tabular}{|c|c|c|c|c|c|c|c|c|c|c|c|c|}
\hline \multirow[b]{2}{*}{ De } & \multicolumn{12}{|c|}{ Para } \\
\hline & PA1 & PB2 & PA3 & PB4 & PA5 & PB6 & PA7 & PB8 & PA9 & PB10 & PA11 & PB12 \\
\hline PA1 & - & 7 & 5 & 12 & 10 & 17 & 15 & 22 & 20 & 27 & 25 & 32 \\
\hline PB2 & 7 & - & 12 & 5 & 17 & 10 & 22 & 15 & 27 & 20 & 32 & 25 \\
\hline PA3 & 5 & 12 & - & 7 & 5 & 12 & 10 & 17 & 15 & 22 & 20 & 27 \\
\hline PB4 & 12 & 5 & 7 & - & 12 & 5 & 17 & 10 & 22 & 15 & 27 & 20 \\
\hline PA5 & 10 & 17 & 5 & 12 & - & 7 & 5 & 12 & 10 & 17 & 15 & 22 \\
\hline PB6 & 17 & 10 & 12 & 5 & 7 & - & 12 & 5 & 17 & 10 & 22 & 15 \\
\hline PA7 & 15 & 22 & 10 & 17 & 5 & 12 & - & 7 & 5 & 12 & 10 & 17 \\
\hline PB8 & 22 & 15 & 17 & 10 & 12 & 5 & 7 & - & 12 & 5 & 17 & 10 \\
\hline PA9 & 20 & 27 & 15 & 22 & 10 & 17 & 5 & 12 & - & 7 & 5 & 12 \\
\hline PB10 & 27 & 20 & 22 & 15 & 17 & 10 & 12 & 5 & 7 & - & 12 & 5 \\
\hline PA11 & 25 & 32 & 20 & 27 & 15 & 22 & 10 & 17 & 5 & 12 & - & 7 \\
\hline PB12 & 32 & 25 & 27 & 20 & 22 & 15 & 17 & 10 & 12 & 5 & 7 & - \\
\hline
\end{tabular}

Por sua vez, a Tabela 5 traz os tempos de residência das pilhas no pátio, também representados em minutos.

Tabela 5 - Tempo de residência das pilhas em minutos

\begin{tabular}{|c|c|c|c|c|c|c|c|c|c|c|c|c|}
\hline Pilha & PA1 & PB2 & PA3 & PB4 & PA5 & PB6 & PA7 & PB8 & PA9 & PB10 & PA11 & PB12 \\
\hline $\mathrm{t}(\mathrm{min})$ & 4.320 & 5.472 & 1.440 & 7.344 & 7.056 & 4.320 & 720 & 2.880 & 8.640 & 4.032 & 2.880 & 1.296 \\
\hline
\end{tabular}

Por fim, a tabela 6 compila as informações de todas as pilhas analisadas, juntamente com os índices de prioridade estimados através da equação (6)

Tabela 6 - Cálculo do índice de priorização de pilhas.

\begin{tabular}{|c|c|c|c|c|c|c|}
\hline & & $\mathbf{Q} i$ & $\mathbf{T} i$ & $\mathbf{S} i$ & IBP $i$ & IP $i$ \\
\hline \multirow{5}{*}{ 吾 } & PA5 & - & - & - & - & - \\
\hline & PA1 & 1 & 4.320 & 10 & 0,563 & 243,3 \\
\hline & $\mathrm{PA}_{3}$ & 5,10 & 1.440 & 5 & 0,563 & 827,3 \\
\hline & PB2 & 9,94 & 5.472 & 17 & 0,437 & $1.396,9$ \\
\hline & PB4 & 9,04 & 7.344 & 12 & 0,437 & $2.416,2$ \\
\hline \multirow{7}{*}{ 晃 } & PB12 & - & - & - & - & - \\
\hline & PA7 & 1 & 720 & 17 & 0,391 & 16,6 \\
\hline & PA9 & 9,60 & 8.640 & 12 & 0,391 & $2.702,2$ \\
\hline & PA11 & 1 & 2.880 & 7 & 0,391 & 160,8 \\
\hline & PB6 & 1 & 4.320 & 15 & 0,609 & 175,4 \\
\hline & PB8 & 9,98 & 2.880 & 10 & 0,609 & $1.749,7$ \\
\hline & PB10 & 3,28 & 4.032 & 5 & 0,609 & $1.609,0$ \\
\hline
\end{tabular}

As pilhas $P_{A} 5$ e $P_{B} 12$ foram escolhidas arbitrariamente como pilhas de partida do modelo, ou seja, pilhas que estão sendo recuperadas e de onde partirão as máquinas recuperadoras. Desta forma, a ordem de recuperação das pilhas para $\mathrm{M} 1$ é $P_{A} 5, P_{B} 4, P_{B} 2, P_{A} 3$ e $P_{A} 1$; para M2 é $P_{B} 12, P_{A} 9, P_{B} 8, P_{B} 10, P_{B} 6, P_{A} 11$ e $P_{A} 7$.

\section{CONCLUSÃO}

A sequência sugerida pela sistemática proposta foi considerada satisfatória pelos especialistas de processo, pois sugeriu que a máquina 2 partisse da pilha $P_{B} 12$ e então para a pilha $P_{A} 9(\mathrm{IP} i=2.702,2)$, ou seja, para uma pilha mais afastada no pátio e com qualidade inferior se comparada com a pilha $P_{B} 8$, com IPi $=1.749,7$. Tal 
escolha, no entanto, justifica-se pelo fato da pilha $P_{A} 9$ estar com um tempo de residência três vezes maior do que a pilha $P_{B} 8$, o que encontra respaldo em critérios práticos.

A proposição desta heurística também contribui academicamente, pois verifica-se uma carência de estudos sobre sequenciamento voltados para operações de pátio de estocagem, formação de pilhas (empilhamento) e recuperação destas para o carregamento de vagões.

Para futuras pesquisas na área de pátios de estocagem de minérios, sugere-se a criação de uma heurística que considere dependência entre as recuperadoras no pátio. Esta configuração traz grandes benefícios em termos de flexibilidade operacional: por exemplo, uma pilha com qualidade inferior à especificação (que não teria alta prioridade no método aqui proposto) passa a ser recuperada juntamente com outra pilha com qualidade acima das especificações e, desta forma, a qualidade do blending final não seja comprometida.

\section{REFERÊNCIAS}

1 Bley, A., Boland, N., Fricke, C., and Floyland, G. (2010). A strengthened formulation and cutting planes for the open pit mine production scheduling problem. Computers \& Operations Research, 37, 1641-1647.

2 Boland, N., Dumitrescu, Floyland, G., and Gleixner, A. M. (2009). LP-based disaggregation approaches to solving the open pit mining production scheduling problem with block processing selectivity. Computers \& Operations Research, 36, 1064-1089.

3 Davis. M. M.; Aquilano, N. J.; Chase, R. B. Fundamentos de Administração da Produção. 3. ed. São Paulo: Bookman. Porto Alegre, 2001.

4 Dronkers, J., and J. T. F. Zimmerman. Some principles of mixing in tidal lagoons. Oceanologia acta. Proceedings of the International Symposium on Coastal Lagoons, Bordeaux, France, 1982.

5 Gilio, N. C. Método heuristico para o problema de no-wait flow shop com setup separado e independente da sequencia. Trabalho de Conclusão de Curso (Graduação em Engenharia de Produção Mecânica) - Escola de Engenharia de São Carlos, Universidade de São Paulo, São Carlos. 2007.

6 Kackar, R. N., Taguchi's Quality Philosophy: Analysis and Commentary. Quality Progress, p. 21-29, New York, 1986.

7 Moura, Reinaldo A., Série Manual de Logística. Volume 2. São Paulo: IMAM,1997.

8 Pinedo, Michael, 2008. Scheduling: Theory, Algorithms, and Systems $-3^{\text {a }}$ ed., New Jersey, Prentice Hall.

9 Taguchi, G., "Off-line and On-line Quality Control Systems." Proceedings of the International Conference on Quality Control, B4, Tokyo, Japan, 1978.

10 VALE. Sistema Norte - Processos das Minas de Ferro e Manganês e Atividades do Turno GADAN. 2008. Relatório interno 